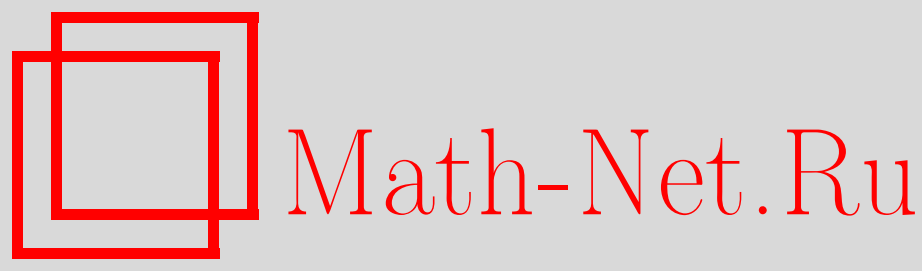

В. В. Саргсян, 3. Каноков, Г. Г. Адамян, Н. В. Антоненко, Квантовые немарковские уравнения Ланжевена и транспортные коэффициенты для перевернутого осциллятора, ТМФ, 2008, том 156, номер 3, 425-443

DOI: https://doi.org/10.4213/tmf6257

Использование Общероссийского математического портала Math-Net.Ru подразумевает, что вы прочитали и согласны с пользовательским соглашением http://www.mathnet.ru/rus/agreement

Параметры загрузки:

IP : 3.89 .197 .203

26 апреля 2023 г., 18:03:02

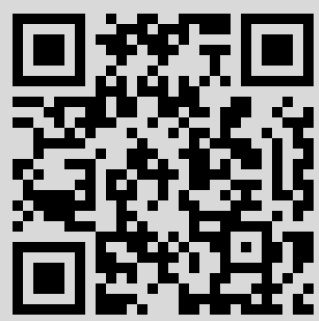




\title{
КВАНТОВЫЕ НЕМАРКОВСКИЕ УРАВНЕНИЯ ЛАНЖЕВЕНА И ТРАНСПОРТНЫЕ КОЭФФИЦИЕНТЫ ДЛЯ ПЕРЕВЕРНУТОГО ОСЦИЛЛЯТОРА
}

\begin{abstract}
На основе немарковских квантовых уравнений Ланжевена получены зависящие от времени транспортные коэффициенты для перевернутого осциллятора, связанного линейно по координате с термостатом. Проведен сравнительный анализ диффузионных коэффициентов гармонического и перевернутого осцилляторов. Изучена роль квантовых статистических эффектов при прохождении через параболический барьер.
\end{abstract}

Ключевые слова: открытые квантовые системы, коэффициенты трения и диффузии, немарковская динамика.

\section{1. ВВЕДЕНИЕ}

Квантовым статистическим эффектам в открытых системах посвящено большое число работ [1]-[16]. Такие эффекты проявляются, например, при слиянии тяжелых ядер, в реакциях многонуклонных передач с тяжелыми ионами и при делении ядер. Эти процессы описываются с помощью диффузионных уравнений в пространстве релевантных коллективных координат. Поскольку диссипация и флуктуации в коллективной квантовой системе возникают вследствие взаимодействия системы с внешней средой (термостатом или внутренними степенями свободы), их полное описание дано на языке матрицы плотности, квантового диффузионного уравнения для функции Вигнера или квантовых уравнений Ланжевена. В кинетической теории метод Ланжевена значительно упрощает вычисление неравновесных квантовых и тепловых флуктуаций и обеспечивает ясную картину динамики процесса [3], [5], [7]-[9], [11], [13], [15]. Во многих исследованиях на основе транспортных моделей квантовые статистические эффекты игнорируются и используется классическое

* Объединенный институт ядерных исследований, г. Дубна, Московская обл., Россия. E-mail: adamian@theor.jinr.ru, antonenk@theor.jinr.ru

${ }^{\dagger}$ Национальный университет Узбекистана им. М. Улугбека, Ташкент, Узбекистан

${ }^{\ddagger}$ Институт ядерной физики АН Республики Узбекистан, Ташкент, Узбекистан 
описание, в котором коэффициенты трения и диффузии связаны через классическое флуктуационно-диссипационное соотношение.

Изучение затухания и флуктуаций в коллективной квантовой системе в основном ограничивалось марковским пределом и пределом слабой связи или высоких температур. Нелокальность диссипации обычно не принималась во внимание при описании достаточно быстрых процессов, например при описании столкновения тяжелых ионов. Как правило, коллективная система представлялась гармоническим осциллятором, взаимодействие которого с термостатом (набором гармонических осцилляторов) осуществляется через линейную связь между координатами. Мало внимания уделялось затухающему квантовому перевернутому осциллятору в квантово-механическом термостате. До сих пор нет модели, которая учитывала бы все квантовые эффекты и эффекты немарковости при прохождении через потенциальный барьер. Процесс прохождения через параболический барьер играет важную роль в химии и физике, например при захвате налетающего ядра ядром мишени при энергиях около кулоновского барьера.

Целью данной работы является получение нестационарных, включающих немарковские эффекты, коэффициентов трения и диффузии для квантового перевернутого осциллятора в случае произвольного затухания и расчет проницаемости потенциального барьера с использованием этих коэффициентов. Специально исследуется случай линейной связи по координате между коллективной подсистемой и термостатом. Как указывалось ранее в работах [9], [12], [15], при такой связи возникает трение и диффузия по коллективному импульсу. В качестве отправной точки используются уравнения Ланжевена, которые получены в разделе 2. Проверено, что эти уравнения удовлетворяют флуктуационно-диссипационному соотношению. Из сравнения уравнений движения для средних и дисперсий с их классическими аналогами выводятся выражения для зависящих от времени коэффициентов трения и диффузии. Полученные аналитические формулы использованы в разделе 3 для анализа диффузионных коэффициентов нормального и перевернутого осцилляторов и описания процесса прохождения через потенциальный параболический барьер.

\section{2. КВАНТОВАЯ НЕМАРКОВСКАЯ ДИНАМИКА}

2.1. Уравнения Ланжевена. Для квантовых ядерных систем в работах [4], [17] был построен квантовый гамильтониан $H$, зависящий явно от коллективной координаты $q$, канонически сопряженного коллективного импульса $p$ и внутренних степеней свободы. Предполагая, что связь коллективной подсистемы с термостатом осуществляется через коллективную координату $q$ и внутренние координаты $q_{\nu}$, полный гамильтониан системы можно записать в следующем виде:

$$
\begin{gathered}
H=H_{\mathrm{c}}+H_{\mathrm{b}}+H_{\mathrm{cb}}, \\
H_{\mathrm{c}}=\frac{1}{2 \mu} p^{2}+U(q), \quad H_{\mathrm{b}}=\sum_{\nu} \hbar \omega_{\nu} b_{\nu}^{+} b_{\nu}, \\
H_{\mathrm{cb}}=\frac{\kappa}{\hbar} \lambda^{1 / 2} q \sum_{\nu} \Gamma_{\nu}\left(b_{\nu}^{+}+b_{\nu}\right)+\frac{\kappa^{2}}{\hbar^{2}} \lambda q^{2} \sum_{\nu} \frac{\left|\Gamma_{\nu}\right|^{2}}{\hbar \omega_{\nu}} .
\end{gathered}
$$


Здесь $U(q)= \pm \mu \omega^{2} q^{2} / 2=\tilde{\delta} q^{2} / 2$ - потенциал коллективной подсистемы, $\tilde{\delta}$ - коэффициент жесткости, знак “+" соответствует случаю, когда коллективная система является гармоническим осциллятором, а знак "-" - перевернутым осциллятором. Операторы $b_{\nu}^{+}$и $b_{\nu}$ - фононные операторы рождения и уничтожения соответственно, описывающие внутренние возбуждения системы с энергией $\hbar \omega_{\nu}$. Операторы $H_{\mathrm{c}}$ и $H_{\mathrm{b}}$ являются гамильтонианами коллективной и внутренней подсистем соответственно. Первый член в $H_{\mathrm{cb}}$ описывает связь коллективного движения с внутренними возбуждениями и является источником появления диссипативных членов в уравнениях для операторов коллективных переменных. Например, при описании взаимодействия ядер при низких энергиях данный член отвечает воздействию среднего поля каждого из ядер на одночастичное движение в другом ядре. Величины $\Gamma_{\nu}-$ константы связи коллективной подсистемы с внутренними координатами $q_{\nu}, \lambda$ - параметр, который определяет среднюю силу взаимодействия с термостатом, $\kappa=(2 \mu \omega \lambda / \hbar)^{1 / 2}$. Дополнительный член в $H_{\mathrm{cb}}$ компенсирует перенормировку потенциала, возникающую из-за связи коллективной и внутренней подсистем [4]. Наша цель - получить и аналитически решить уравнения Ланжевена для коллективных операторов $p$ и $q$. Квадратичный гамильтониан допускает точное решение уравнений движения для коллективных координат.

Используя гамильтониан (1), получаем систему уравнений Гейзенберга для операторов, относящихся к коллективному и внутреннему движениям:

$$
\begin{aligned}
& \dot{q}=\frac{i}{\hbar}[H, q]=\frac{1}{\mu} p, \\
& \dot{p}=\frac{i}{\hbar}[H, p]=\mp \mu \omega^{2} q-q \sum_{\nu} \Gamma_{\nu}\left(b_{\nu}^{+}+b_{\nu}\right), \\
& \dot{b}_{\nu}^{+}=\frac{i}{\hbar}\left[H, b_{\nu}^{+}\right]=i \omega_{\nu} b_{\nu}^{+}+i \frac{\kappa}{\hbar^{2}} \lambda^{1 / 2} q \Gamma_{\nu}, \\
& \dot{b}_{\nu}=\frac{i}{\hbar}\left[H, b_{\nu}\right]=-i \omega_{\nu} b_{\nu}-i \frac{\kappa}{\hbar^{2}} \lambda^{1 / 2} q \Gamma_{\nu} .
\end{aligned}
$$

Учет влияния коллективной подсистемы на термостат необходим, так как это влияние является причиной появления диссипативного члена в уравнениях движения коллективной подсистемы. При $t>0$ степени свободы термостата эволюционируют согласно уравнениям (3) и термостат отклоняется от начального $(t=0)$ состояния теплового равновесия. Когда влияние коллективной подсистемы на термостат игнорируется, диссипативное ядро равно нулю и термостат всегда находится в состоянии теплового равновесия.

Подставляя решения уравнений (3) в уравнения (2), получаем систему интегродифференциальных стохастических уравнений [15]

$$
\begin{gathered}
\dot{q}(t)=\frac{p(t)}{\mu}, \\
\dot{p}(t)=-\tilde{\delta} q(t)-\kappa^{2} \int_{0}^{t} d \tau K(t-\tau) \dot{q}(\tau)+\kappa F(t),
\end{gathered}
$$


которую называют системой обобщенных нелинейных уравнений Ланжевена. Присутствие интегрального члена в уравнениях движения означает, что система немарковская и обладает “памятью” о движении по траектории, предшествующей моменту времени $t$. Как видно из уравнения для $p(t)$, наличие связи по координате приводит к появлению случайной силы по импульсу

$$
\begin{gathered}
F(t)=\frac{F_{p}(t)}{\kappa}=\sum_{\nu} F^{\nu}(t)=-\frac{\lambda^{1 / 2}}{\hbar} \sum_{\nu} \Gamma_{\nu}\left[f_{\nu}^{+}(t)+f_{\nu}(t)\right], \\
f_{\nu}^{+}(t)=\left[b_{\nu}^{+}(0)+\frac{1}{\hbar^{2} \omega_{\nu}} \kappa \lambda^{1 / 2} \Gamma_{\nu} q(0)\right] e^{i \omega_{\nu} t},
\end{gathered}
$$

и диссипативного ядра

$$
K(t-\tau)=\frac{2 \lambda}{\hbar^{2}} \sum_{\nu} \frac{\Gamma_{\nu}^{2}}{\hbar \omega_{\nu}} \cos \left[\omega_{\nu}(t-\tau)\right]
$$

Как видно из формул (5) и (6), случайная сила и диссипативное ядро не зависят от динамических $(t>0)$ координат и импульсов коллективной и внутренней подсистем. Температура и флуктуации задаются с помощью распределения начальных $(t=0)$ координат и импульсов внутренней системы. Операторы случайных сил $F^{\nu}$ отождествляются с флуктуациями из-за неопределенности начальных условий для операторов термостата. Для определения статистических свойств этих флуктуаций рассмотрим ансамбль начальных состояний, в котором задана начальная коллективная координата $q(0)$, а начальные операторы термостата выбираются из канонического ансамбля [11]. В этом ансамбле флуктуации $F^{\nu}(t)$ имеют вид распределения Гаусса с нулевым средним значением

$$
\left\langle\left\langle F^{\nu}(t)\right\rangle\right\rangle=0
$$

и ненулевой дисперсией. Символ 〈〈...〉 обозначает среднее по переменным термостата. Нормальное распределение случайных сил соответствует случаю, когда термостат представляется набором гармонических осцилляторов [3], [5], [9], [11], [12], [15]. В настоящей работе для расчета корреляционных функций флуктуаций используется термостат, подчиняющийся статистике Бозе-Эйнштейна:

$$
\begin{aligned}
& \left\langle\left\langle f_{\nu}^{+}(t) f_{\nu^{\prime}}^{+}\left(t^{\prime}\right)\right\rangle\right\rangle=\left\langle\left\langle f_{\nu}(t) f_{\nu^{\prime}}\left(t^{\prime}\right)\right\rangle\right\rangle=0, \\
& \left\langle\left\langle f_{\nu}^{+}(t) f_{\nu^{\prime}}\left(t^{\prime}\right)\right\rangle\right\rangle=\delta_{\nu, \nu^{\prime}} n_{\nu} e^{i \omega_{\nu}\left(t-t^{\prime}\right)}, \\
& \left\langle\left\langle f_{\nu}(t) f_{\nu^{\prime}}^{+}\left(t^{\prime}\right)\right\rangle\right\rangle=\delta_{\nu, \nu^{\prime}}\left(n_{\nu}+1\right) e^{-i \omega_{\nu}\left(t-t^{\prime}\right)},
\end{aligned}
$$

где $n_{\nu}=\left[e^{\hbar \omega_{\nu} / T}-1\right]^{-1}-$ температурные числа заполнения для фононов, $T$ - температура термостата.

Используя статистические свойства (7), (8) случайной силы (5), получаем следующее соотношение для симметризованной корреляционной функции случайной силы:

$$
\phi^{\nu}\left(t-t^{\prime}\right)=\left\langle\left\langle F^{\nu}(t) F^{\nu}\left(t^{\prime}\right)+F^{\nu}\left(t^{\prime}\right) F^{\nu}(t)\right\rangle\right\rangle=\left(2 n_{\nu}+1\right) \frac{2 \lambda}{\hbar^{2}} \frac{\Gamma_{\nu}}{\hbar \omega_{\nu}} \cos \left[\omega_{\nu}\left(t-t^{\prime}\right)\right] .
$$


Используя выражение для диссипативного ядра (6) и учитывая, что $2 n_{\nu}+1=$ $\operatorname{cth}\left[\hbar \omega_{\nu} / 2 T\right]$, получаем квантовое флуктуационно-диссипационное соотношение

$$
\sum_{\nu} \phi^{\nu}\left(t-t^{\prime}\right) \frac{\operatorname{th}\left[\hbar \omega_{\nu} / 2 T\right]}{\hbar \omega_{\nu}}=K\left(t-t^{\prime}\right) .
$$

В рассмотренном случае связи между коллективной и внутренней подсистемами корреляционная функция случайной силы и диссипативное ядро не зависят от динамических координат и импульсов полной системы. По этой причине флуктуационнодиссипационное соотношение не зависит от динамических чисел заполнения для фононов. Числа заполнения в формулах (8) и в квантовом флуктуационно-диссипационном соотношении относятся к начальному моменту времени, когда термостат находится в состоянии теплового равновесия.

Таким образом, уравнения движения для коллективных координат удовлетворяют квантовому флуктуационно-диссипационному соотношению, которое отличается от классического и сводится к нему в пределе большой температуры $T$ (или $\hbar \rightarrow 0$ ), когда в формуле $(9) \operatorname{th}\left[\hbar \omega_{\nu} / 2 T\right] \rightarrow \hbar \omega_{\nu} / 2 T$. Классические соотношения содержат лишь температурные флуктуации. В (9) дополнительно учитываются еще и квантовые флуктуации. Наш формализм позволяет корректно описывать квантовые статистические эффекты коллективного движения.

Для аналитического решения уравнений (4) применим метод преобразований Лапласа. Получив выражения для образов, находим явные выражения для оригиналов:

$$
\begin{aligned}
& q(t)=A_{t} q(0)+B_{t} p(0)+\kappa \int_{0}^{t} d \tau B_{\tau} F(t-\tau), \\
& p(t)=M_{t} q(0)+N_{t} p(0)+\kappa \int_{0}^{t} d \tau N_{\tau} F(t-\tau),
\end{aligned}
$$

где

$$
\begin{gathered}
B_{t}=\frac{1}{\mu} \mathcal{L}^{-1}\left[\frac{1}{s^{2}+2 \hbar \omega s K(s)+\tilde{\delta} / \mu}\right] \\
A_{t}=\mathcal{L}^{-1}\left[\frac{s+2 \hbar \omega K(s)}{s^{2}+2 \hbar \omega s K(s)+\tilde{\delta} / \mu}\right]=\mu \dot{B}_{t}+\kappa^{2} \int_{0}^{t} d \tau B_{\tau} K(t-\tau), \\
M_{t}=-\mu \tilde{\delta} B_{t}, \quad N_{t}=\mu \dot{B}_{t} .
\end{gathered}
$$

Здесь $\mathcal{L}^{-1}$ обозначает обратное преобразование Лапласа, $K(s)$ - изображение диссипативного ядра $K(t)$. Символы $t$ и $\tau$ указывают на временну́ю зависимость.

2.2. Транспортные коэффициенты. Используя средние значения $q(t)$ и $p(t)$ :

$$
\begin{aligned}
& \langle q(t)\rangle=A_{t}\langle q(0)\rangle+B_{t}\langle p(0)\rangle, \\
& \langle p(t)\rangle=M_{t}\langle q(0)\rangle+N_{t}\langle p(0)\rangle,
\end{aligned}
$$


дифференцируя их по времени и выражая $\langle q(0)\rangle$ и $\langle p(0)\rangle$ через $\langle q(t)\rangle$ и $\langle p(t)\rangle$, получим уравнения для первых моментов

$$
\begin{aligned}
\frac{d}{d t}\langle q(t)\rangle & =\frac{1}{\mu}\langle p(t)\rangle, \\
\frac{d}{d t}\langle p(t)\rangle & =-\xi(t)\langle q(t)\rangle-\lambda_{p}(t)\langle p(t)\rangle .
\end{aligned}
$$

Эти уравнения содержат коэффициент трения по импульсу

$$
\lambda_{p}(t)=\frac{A_{t} \dot{N}_{t}-B_{t} \dot{M}_{t}}{B_{t} M_{t}-A_{t} N_{t}}
$$

и перенормированный коэффициент жесткости

$$
\xi(t)=\frac{\dot{M}_{t} N_{t}-\dot{N}_{t} M_{t}}{B_{t} M_{t}-A_{t} N_{t}} .
$$

Перенормировка потенциала возникает из-за немарковости уравнений движения. В марковском пределе $\xi(t)=\tilde{\delta}$.

Аналогично получив уравнения движения

$$
\begin{aligned}
& \dot{\sigma}_{q q}(t)=\frac{2}{\mu} \sigma_{q p}(t) \\
& \dot{\sigma}_{p p}(t)=-2 \lambda_{p}(t) \sigma_{p p}(t)-2 \xi(t) \sigma_{q p}(t)+2 D_{p p}(t), \\
& \dot{\sigma}_{q p}(t)=-\lambda_{p}(t) \sigma_{q p}(t)-\xi(t) \sigma_{q q}(t)+\frac{1}{\mu} \sigma_{p p}(t)+2 D_{p q}(t)
\end{aligned}
$$

для дисперсий по координате $\sigma_{q q}(t)=\left\langle q^{2}(t)\right\rangle-\langle q(t)\rangle^{2}$, импульсу $\sigma_{p p}(t)=\left\langle p^{2}(t)\right\rangle-$ $\langle p(t)\rangle^{2}$ и координате-импульсу $\sigma_{q p}(t)=\frac{1}{2}\langle p(t) q(t)+q(t) p(t)\rangle-\langle p(t)\rangle\langle q(t)\rangle$, можно определить коэффициенты диффузии по импульсу

$$
\begin{aligned}
D_{p p}(t) & =\lambda_{p}(t) J_{p_{t} p_{t}}+\xi(t) J_{q_{t} p_{t}}+\frac{1}{2} \frac{d}{d t} J_{p_{t} p_{t}}= \\
& =\lambda_{p}(t) J_{p_{t} p_{t}}+\frac{1}{2}\left(\frac{d}{d t} J_{p_{t} p_{t}}+\mu \xi(t) \frac{d}{d t} J_{q_{t} q_{t}}\right)
\end{aligned}
$$

и по координате-импульсу

$$
\begin{aligned}
D_{p q}(t) & =\frac{1}{2}\left[\lambda_{p}(t) J_{q_{t} p_{t}}+\xi(t) J_{q_{t} q_{t}}-\frac{1}{\mu} J_{p_{t} p_{t}}+\frac{d}{d t} J_{q_{t} p_{t}}\right]= \\
& =\frac{1}{2}\left[\xi(t) J_{q_{t} q_{t}}-\frac{1}{\mu} J_{p_{t} p_{t}}+\frac{\mu}{2}\left(\lambda_{p}(t) \frac{d}{d t}+\frac{d^{2}}{d t^{2}}\right) J_{q_{t} q_{t}}\right],
\end{aligned}
$$

где

$$
\begin{aligned}
& J_{q_{t} q_{t}}=\frac{2 \omega \mu \lambda}{\hbar} \sum_{\nu}\left|\Gamma_{\nu}\right|^{2} \operatorname{cth}\left[\frac{\hbar \omega_{\nu}}{2 T}\right] \int_{0}^{t} d \tau^{\prime} B_{\tau^{\prime}} \int_{0}^{t} d \tau^{\prime \prime} B_{\tau^{\prime \prime}} \cos \left[\omega_{\nu}\left(\tau^{\prime}-\tau^{\prime \prime}\right)\right] \\
& J_{p_{t} p_{t}}=\frac{2 \omega \mu \lambda}{\hbar} \sum_{\nu}\left|\Gamma_{\nu}\right|^{2} \operatorname{cth}\left[\frac{\hbar \omega_{\nu}}{2 T}\right] \int_{0}^{t} d \tau^{\prime} N_{\tau^{\prime}} \int_{0}^{t} d \tau^{\prime \prime} N_{\tau^{\prime \prime}} \cos \left[\omega_{\nu}\left(\tau^{\prime}-\tau^{\prime \prime}\right)\right], \\
& J_{q_{t} p_{t}}=\frac{2 \omega \mu \lambda}{\hbar} \sum_{\nu}\left|\Gamma_{\nu}\right|^{2} \operatorname{cth}\left[\frac{\hbar \omega_{\nu}}{2 T}\right] \int_{0}^{t} d \tau^{\prime} N_{\tau^{\prime}} \int_{0}^{t} d \tau^{\prime \prime} B_{\tau^{\prime \prime}} \cos \left[\omega_{\nu}\left(\tau^{\prime}-\tau^{\prime \prime}\right)\right] .
\end{aligned}
$$


Таким образом, мы получили нелинейные уравнения на первые и вторые моменты с транспортными коэффициентами, зависящими явно от времени. Именно зависимость коэффициентов от времени является следствием немарковости системы. За появление в стохастических уравнениях коэффициентов трения $\lambda_{p}$ и диффузии $D_{p p}, D_{p q}$ ответственна линейная связь по координате $q$.

Используя теорему о вычетах для нахождения оригиналов, решения $q(t)$ и $p(t)$ в (10) выразим через корни $s_{i}, i=1,2,3$, уравнения

$$
d(s)=s^{2}+2 \hbar \omega s K(s)+\frac{\tilde{\delta}}{\mu}=0 .
$$

Для этого определим явный вид диссипативного ядра следующим образом. Записав сумму $\sum_{\nu} \ldots$ в виде интеграла $\int_{0}^{\infty} d \omega_{0} g\left(\omega_{0}\right) \ldots$ по частоте с плотностью состояний $g\left(\omega_{0}\right)$ термостата [11], [15], где

$$
\frac{g\left(\omega_{0}\right)\left|\Gamma\left(\omega_{0}\right)\right|^{2}}{\hbar^{2} \omega_{0}}=\frac{\gamma^{2}}{\pi\left(\gamma^{2}+\omega_{0}^{2}\right)},
$$

из соотношения (6) получаем

$$
K(t)=\frac{\lambda \gamma}{\hbar} e^{-\gamma|t|}
$$

Такой выбор плотности состояний оправдан тем, что в предельном случае $\gamma \rightarrow \infty$ мы получаем марковский предел. Подставляя образ $K(s)=\lambda \gamma /(\hbar(s+\gamma))$ диссипативного ядра в (11) и (20), получаем

$$
\begin{aligned}
B_{t} & =\sum_{i=1}^{3} B_{t}^{i}=\frac{1}{\mu} \sum_{i=1}^{3} \beta_{i}\left(s_{i}+\gamma\right) e^{s_{i} t}, \\
A_{t} & =\sum_{i=1}^{3} \beta_{i}\left[s_{i}\left(s_{i}+\gamma\right)+2 \omega \lambda \gamma\right] e^{s_{i} t}, \\
d(s) & =\frac{(s+\gamma)\left(s^{2}+\tilde{\delta} / \mu\right)-2 \omega \lambda \gamma s}{s+\gamma}=0,
\end{aligned}
$$

где $\beta_{1}=1 /\left[\left(s_{1}-s_{2}\right)\left(s_{1}-s_{3}\right)\right], \beta_{2}=1 /\left[\left(s_{2}-s_{1}\right)\left(s_{2}-s_{3}\right)\right], \beta_{3}=1 /\left[\left(s_{3}-s_{1}\right)\left(s_{3}-s_{2}\right)\right]$.

Для асимптотических значений коэффициентов трения, жесткости и диффузии получаем из (14), (15) и (17)-(19) следующие выражения:

$$
\begin{aligned}
\lambda_{p}(\infty) & =-\left(s_{2}+s_{1}\right), \\
\xi(\infty) & =\tilde{\delta} \frac{\left(s_{1}+\gamma\right)\left(s_{2}+\gamma\right)}{\left(s_{1}+\gamma\right)\left(s_{2}+\gamma\right)-2 \lambda \gamma \omega}, \\
D_{p p}(\infty) & =\lambda_{p}(\infty) J_{p_{\infty} p_{\infty}}+\xi(\infty) J_{q_{\infty} p_{\infty}}, \\
D_{p q}(\infty) & =\frac{1}{2}\left[\lambda_{p}(\infty) J_{q_{\infty} p_{\infty}}+\xi(\infty) J_{q_{\infty} q_{\infty}}-\frac{1}{\mu} J_{p_{\infty} p_{\infty}}\right],
\end{aligned}
$$


где

$$
\begin{aligned}
J_{q_{\infty} q_{\infty}}= & \frac{2 \hbar \omega \lambda \gamma^{2}}{\pi \mu} \sum_{i, j} \beta_{i} \beta_{j}\left(s_{i}+\gamma\right)\left(s_{j}+\gamma\right) \phi^{a}\left(s_{i}, s_{j}\right), \\
J_{p_{\infty} p_{\infty}}= & \frac{2 \hbar \omega \mu \lambda \gamma^{2}}{\pi} \sum_{i, j} \beta_{i} \beta_{j} s_{i} s_{j}\left(s_{i}+\gamma\right)\left(s_{j}+\gamma\right) \phi^{a}\left(s_{i}, s_{j}\right), \\
J_{q_{\infty} p_{\infty}}= & \frac{\hbar \omega \lambda \gamma^{2}}{\pi} \sum_{i, j} \beta_{i} \beta_{j}\left(s_{i}+s_{j}\right)\left(s_{i}+\gamma\right)\left(s_{j}+\gamma\right) \phi^{a}\left(s_{i}, s_{j}\right) \\
\phi^{a}\left(s_{i}, s_{j}\right)= & \frac{s_{j} \psi\left(-\hbar s_{j} / 2 \pi T\right)}{\left(s_{i}+s_{j}\right)\left(s_{j}^{2}-\gamma^{2}\right)}+\frac{s_{i} \psi\left(-\hbar s_{i} / 2 \pi T\right)}{\left(s_{i}+s_{j}\right)\left(s_{i}^{2}-\gamma^{2}\right)}+ \\
& +\frac{\left(\gamma^{2}-s_{i} s_{j}\right) \psi(\hbar \gamma / 2 \pi T)}{\left(\gamma^{2}-s_{i}^{2}\right)\left(\gamma^{2}-s_{j}^{2}\right)}-\frac{\pi T\left(s_{i}+s_{j}-2 \gamma\right)}{\hbar \gamma\left(s_{i}+s_{j}\right)\left(\gamma-s_{i}\right)\left(\gamma-s_{j}\right)}
\end{aligned}
$$

В выражениях (23)-(27) $s_{1}$ и $s_{2}$ - сопряженные корни уравнения (20). В случае трех действительных корней берутся два корня, для которых сумма $s_{i}+s_{j}$ максимальна. В выражениях (27) функция $\psi(z)=\Gamma^{\prime}(z) / \Gamma(z)$ является логарифмической производной гамма-функции. В случае гармонического осциллятора выражения для $J_{i_{\infty}} j_{\infty}$ можно упростить:

$$
\begin{aligned}
J_{q_{\infty} q_{\infty}} & =\frac{2 \hbar \omega \lambda \gamma^{2}}{\pi \mu} \int_{0}^{\infty} d \omega_{0} \frac{\omega_{0} \operatorname{cth}\left[\hbar \omega_{0} / 2 T\right]}{\left(s_{1}^{2}+\omega_{0}^{2}\right)\left(s_{2}^{2}+\omega_{0}^{2}\right)\left(s_{3}^{2}+\omega_{0}^{2}\right)}, \\
J_{p_{\infty} p_{\infty}} & =\frac{2 \hbar \omega \mu \lambda \gamma^{2}}{\pi} \int_{0}^{\infty} d \omega_{0} \frac{\omega_{0}^{3} \operatorname{cth}\left[\hbar \omega_{0} / 2 T\right]}{\left(s_{1}^{2}+\omega_{0}^{2}\right)\left(s_{2}^{2}+\omega_{0}^{2}\right)\left(s_{3}^{2}+\omega_{0}^{2}\right)}, \\
J_{q_{\infty} p_{\infty}} & =0 .
\end{aligned}
$$

\section{3. ЧИСЛЕННЫЕ РАСЧЕТЫ И ОБСУЖДЕНИЕ РЕЗУЛЬТАТОВ}

\section{1. Диффузионные коэффициенты гармонического и перевернутого} осцилляторов. Коэффициенты диффузии и трения зависят от параметров $\mu, \omega, \lambda$ и $\gamma$. По определению значение $\gamma$ должно быть много больше $\omega$. Будем использовать следующие значения параметров: $\hbar \gamma=12 \mathrm{MэB} \mathrm{и} \mu=448 \mu_{0}$, где $\mu_{0}$ - масса нуклона. Параметры $\omega$ и $\lambda$ выбираются так, чтобы асимптотические коэффициенты жесткости и трения имели определенные фиксированные значения $\xi(\infty)=\xi= \pm \mu \widetilde{\omega}^{2}$ и $\lambda_{p}(\infty)=\lambda_{p}$. Для сравнения результатов, полученных для гармонического осциллятора и перевернутого осциллятора, параметры $(\gamma, \mu,|\xi(\infty)|)$ для них задавались одинаковыми. В расчетах проницаемости использованы следующие начальные значения параметров гауссовского пакета: $\sigma_{q q}(0)=0.046 \phi_{\mathrm{m}^{2}}, \sigma_{p p}(0)=\hbar^{2} /\left(4 \sigma_{q q}(0)\right)$ $\hbar^{2} \phi м^{-2}, \sigma_{q p}(0)=0, q_{0}=-1$ фм и $p_{0}=7.2 \hbar \phi м^{-1}$.

При $\hbar \widetilde{\omega}=1$ МэВ зависимости микроскопических коэффициентов $\lambda_{p}(t), D_{p p}(t)$ и $D_{p q}(t)$ от времени приведены на рис. 1 для перевернутого осциллятора и на рис. 2 для гармонического осциллятора. Как видно из рисунков, транспортные коэффициенты равны нулю при $t=0$ и через короткое время порядка $\sim 1 / \gamma$ они достигают своих асимптотических значений. Из сравнения рис. 1 и рис. 2 видно, что при относительно высоких температурах $(T=1 \mathrm{MэB})$ асимптотические значения $D_{p p}(\infty)$ и 


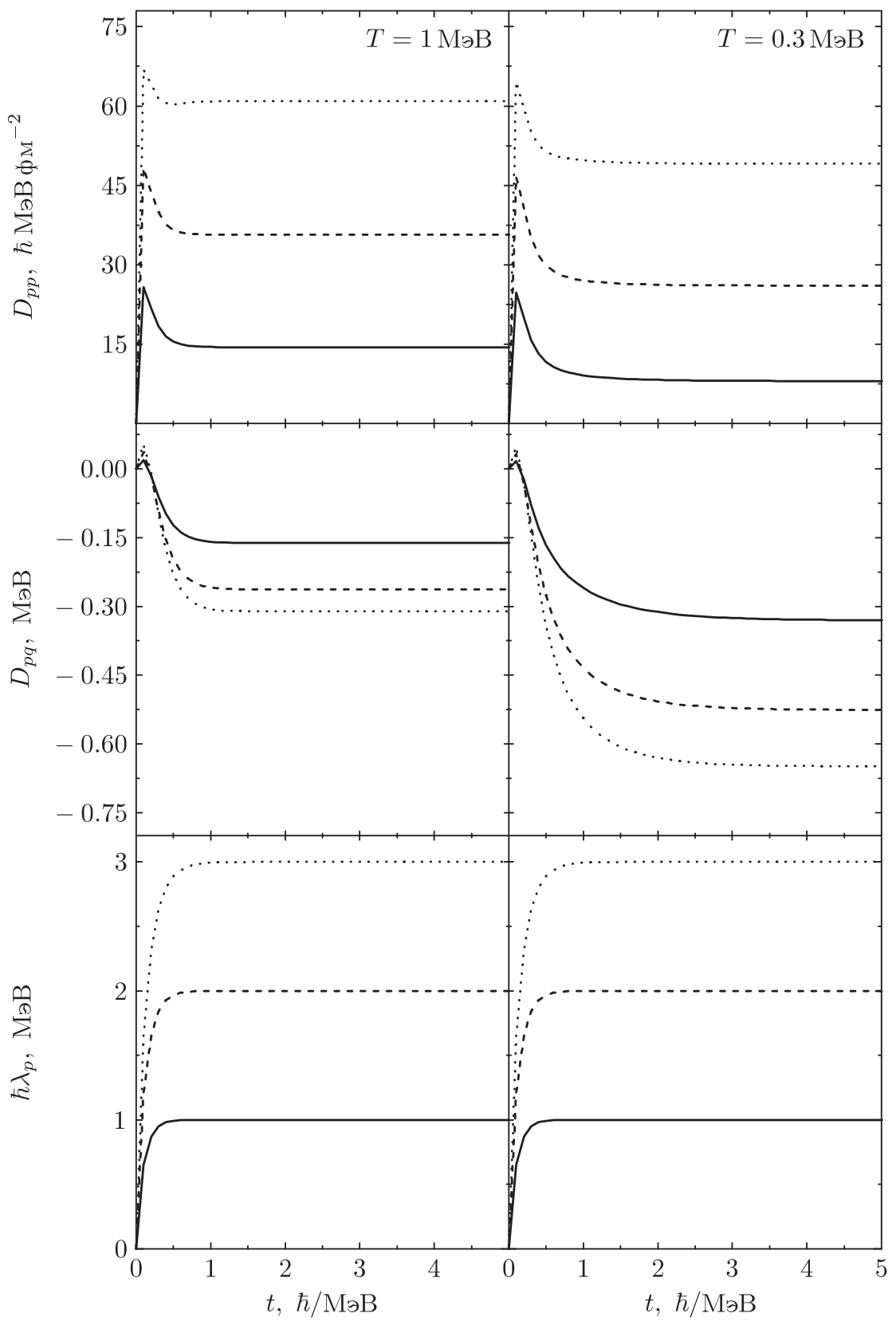

Рис. 1. Зависимости от времени коэффициентов диффузии (17)-(19) и трения (14) для перевернутого осциллятора $\left(\mu=448 m_{0}\right.$ и $\hbar \widetilde{\omega}=1 \mathrm{MэВ)} \mathrm{при}$ $T=1$ МэВ (слева) и $T=0.3$ МэВ (справа). Асимптотики коэффициента трения $\hbar \lambda_{p}=1,2$ и 3 МэВ показаны сплошной, штриховой и пунктирной линиями соответственно. 


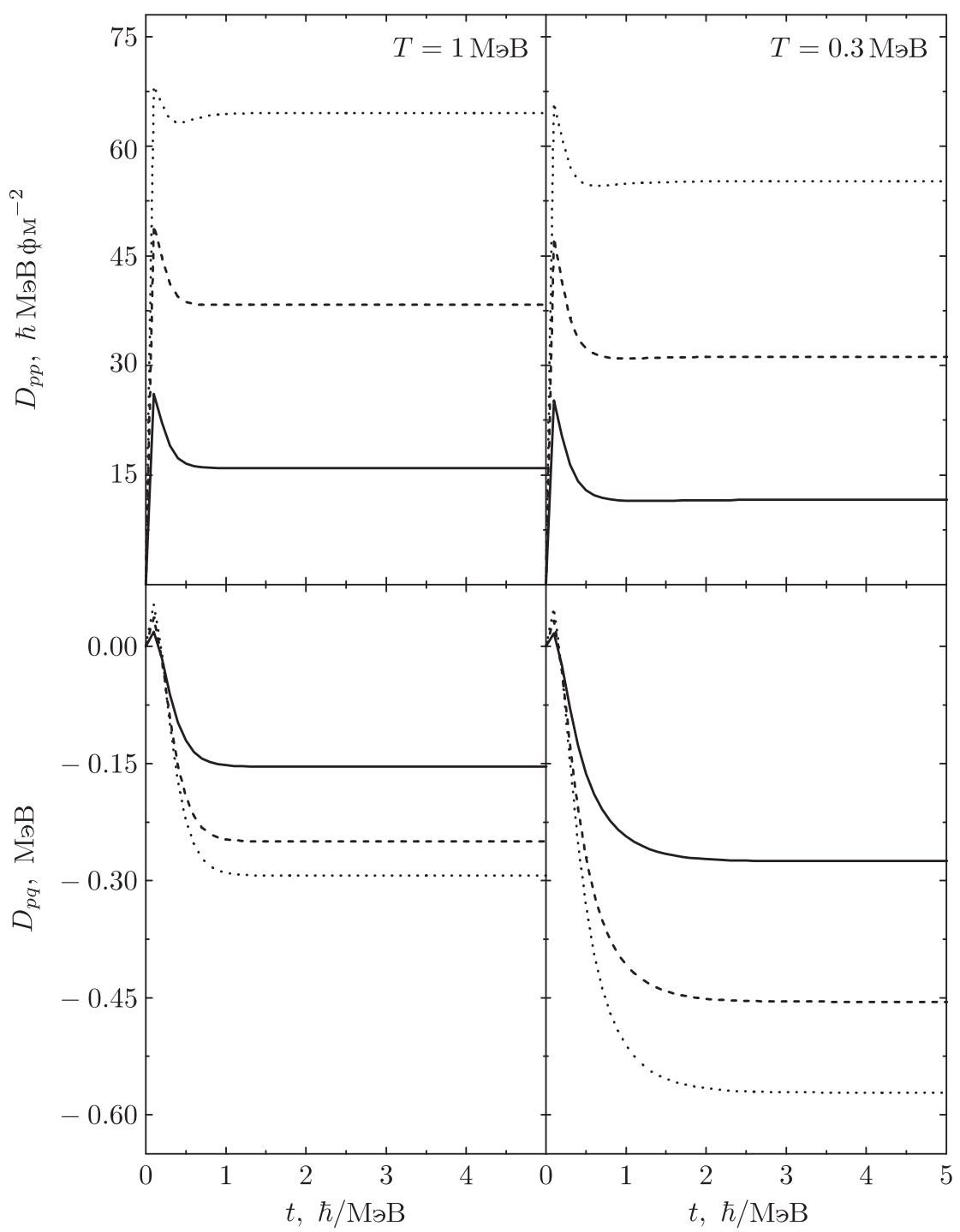

Рис. 2. Зависимости от времени коэффициентов диффузии для гармонического осциллятора. Зависимости $\lambda_{p}(t)$ совпадают с соответствующими зависимостями на рис. 1. 
$D_{p q}(\infty)$ для перевернутого осциллятора приблизительно на $5 \%$ меньше, чем для гармонического осциллятора. При температуре $T=0.3 \mathrm{MэB} \mathrm{значения} D_{p p}(\infty)$ $\left(D_{p q}(\infty)\right)$ для гармонического осциллятора больше на $40 \%(20 \%), 16 \%(15 \%)$ и $11 \%$ $(13 \%)$, чем для перевернутого осциллятора при значениях $\hbar \lambda_{p}(\infty)=1,2$ и 3 МэВ соответственно. Этот диапазон значений коэффициента трения охватывает режимы слабого, среднего и сильного затуханий. Для сравнения с асимптотическими значениями коэффициента диффузии $D_{p p}(\infty)$ на рис. 3 приведена также зависимость от $\lambda_{p}(\infty)$ "классического" феноменологического коэффициента диффузии по импульсу

$$
D_{p p}^{\mathrm{cl}}=\lambda_{p} \frac{\hbar \widetilde{\omega}}{2} \operatorname{cth}\left(\frac{\hbar \widetilde{\omega}}{2 T}\right)
$$

При относительно малых трениях $\hbar \lambda_{p} \leqslant 1$ МэВ асимптотики микроскопических коэффициентов диффузии $D_{p p}(\infty)$ для гармонического осциллятора и перевернутого осциллятора близки к феноменологическому коэффициенту диффузии $D_{p p}^{\mathrm{cl}}$. C увеличением величины коэффициента трения от $\hbar \lambda_{p}=1 \mathrm{MэB} \mathrm{до} \hbar \lambda_{p}=3 \mathrm{MэB} \mathrm{различие}$ достигает коэффициента 2 для высоких температур $(T=1$ МэВ) и коэффициента 3 для низких температур $(T=0.3 \mathrm{MэB})$, причем всегда $D_{p p}(\infty)>D_{p p}^{\mathrm{cl}}$. В отличие от $D_{p p}$, коэффициент $D_{p q}$ не имеет своего классического аналога. Зависимости $D_{p q}$ от $\lambda_{p}$ приведены на рис. 3 .

На рис. 4 показаны изменения $D_{p p}(\infty)$ и $D_{p q}(\infty)$ при переходе от гармонического осциллятора к перевернутому осциллятору, что соответствует изменению знака коэффициента жесткости $\xi$. Из рис. 4 видно, что асимптотические значения $D_{p p}(\infty)$ и $D_{p q}(\infty)$ меняются непрерывно при пересечении точки $\widetilde{\omega}=0$. В рассмотренном диапазоне частот абсолютные значения $D_{p p}(\infty)$ и $D_{p q}(\infty)$ для перевернутого и гармонического осцилляторов относительно близки при фиксированных частоте и температуре. Различие значений коэффициентов диффузии для разного типа осцилляторов уменьшается с увеличением температуры и уменьшением частоты. При температуре $T=2$ МэВ на рис. 5 диффузионные коэффициенты для гармонического и перевернутого осцилляторов $(\hbar \widetilde{\omega}=1 \mathrm{MэB})$ практически совпадают.

Для перевернутого осциллятора мы сталкиваемся с ограничением при расчете диффузионных коэффициентов по формулам (17)-(19) при низких температурах $T \leqslant T_{\text {кр }}=\hbar s_{+} / 2 \pi$, где $T_{\text {кр }}$ - критическая температура перехода от режима термической активации к режиму макроскопического квантового туннелирования через параболический барьер $\left(s_{3}=s_{+}\right.$- действительный положительный корень секулярного уравнения $(20)): D_{p p}$ и $D_{p q}$ не имеют асимптотики и расходятся как функции времени, а $D_{p p}$ становится отрицательным. На рис. 5 мы видим рост отклонения от гармонического осциллятора с приближением к $T_{\text {кр }}$ со стороны больших температур. Это происходит из-за того, что корреляционное время флуктуаций случайной силы $\tau_{\text {кор }}=\hbar / T$ [11] становится сравнимым или бо́льшим, чем характерное время $\tau_{\text {дин }}=2 \pi / s_{+}$динамического изменения импульса коллективной подсистемы. В марковском пределе

$$
\tau_{\text {дин }}=\frac{2 \pi}{\left[\sqrt{\widetilde{\omega}^{2}+\left(\lambda_{p} / 2\right)^{2}}-\lambda_{p} / 2\right]} .
$$




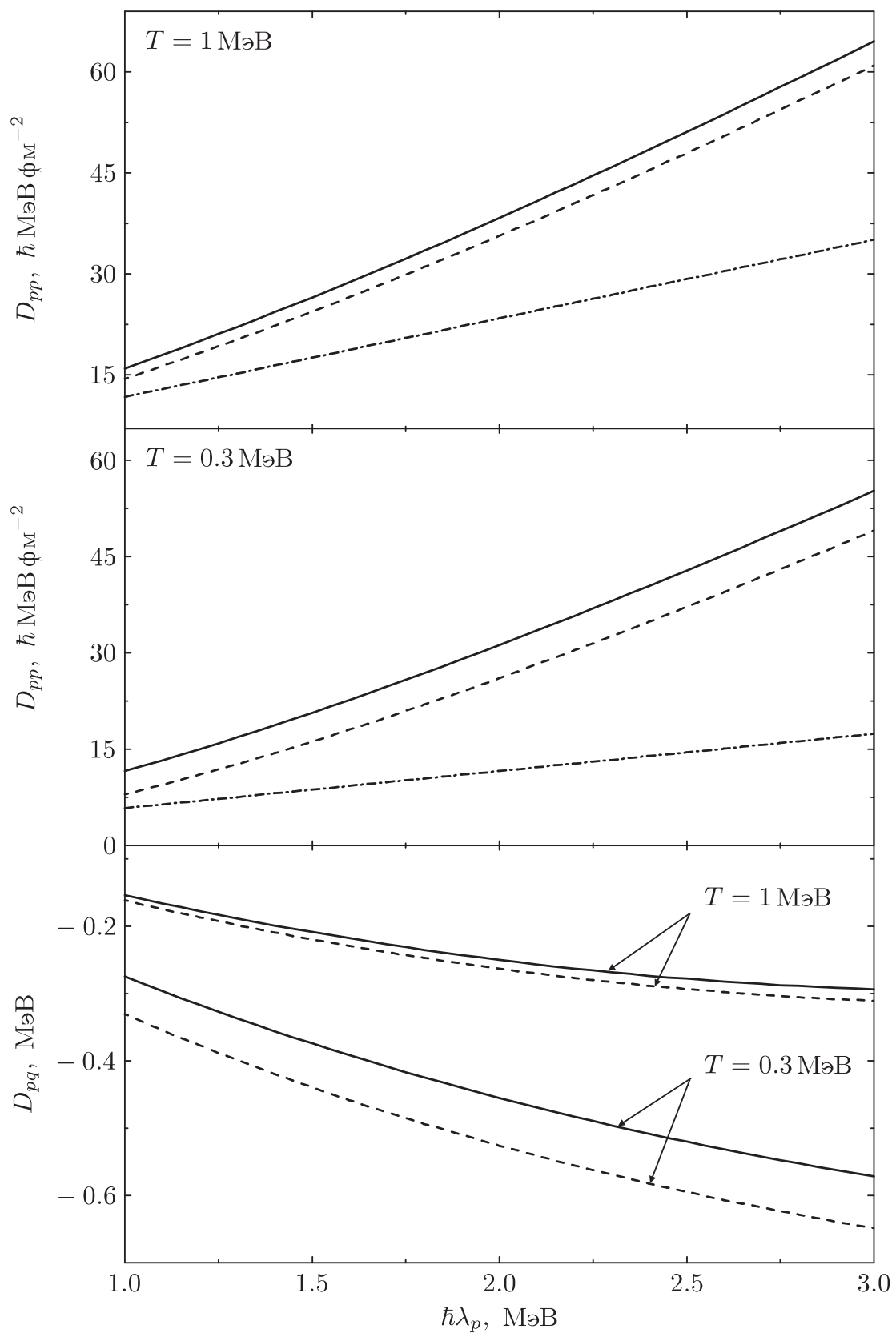

Рис. 3. Зависимости $D_{p p}(\infty)$ и $D_{p q}(\infty)$ от асимптотического значения коэффициента трения при $\hbar \widetilde{\omega}=1 \mathrm{MэB}, \mu=448 m_{0}$. Показаны расчеты по формулам (25)-(27) для гармонического (сплошные линии) и перевернутого (штриховые линии) осцилляторов и по феноменологической формуле (29) (штрихпунктирные линии). 


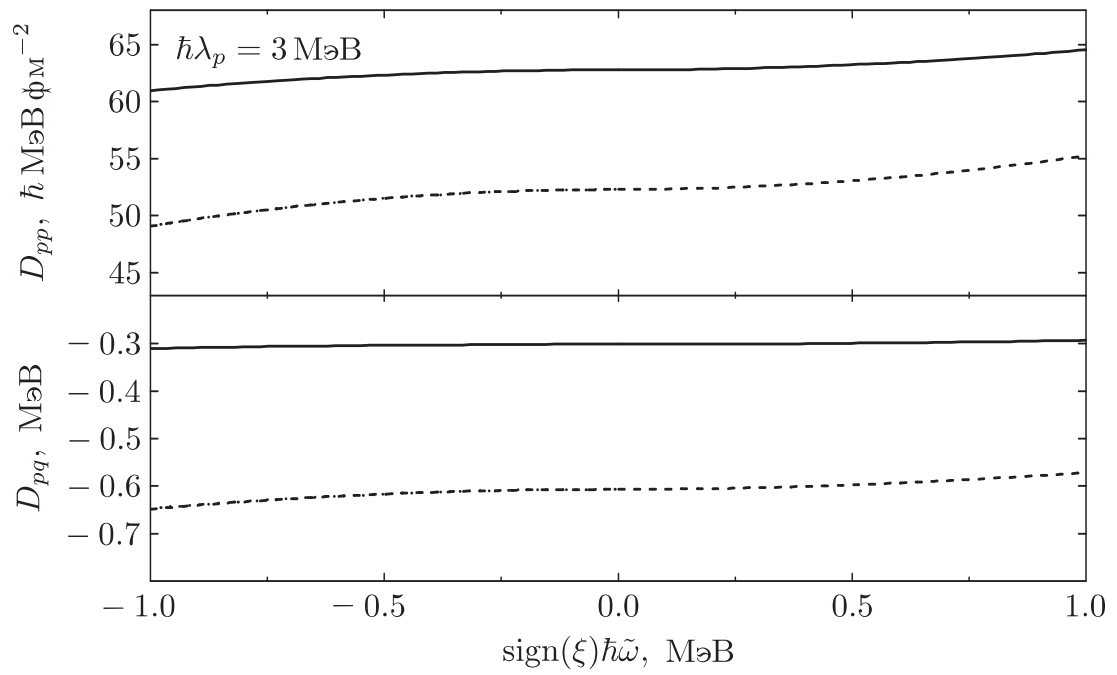

$a$

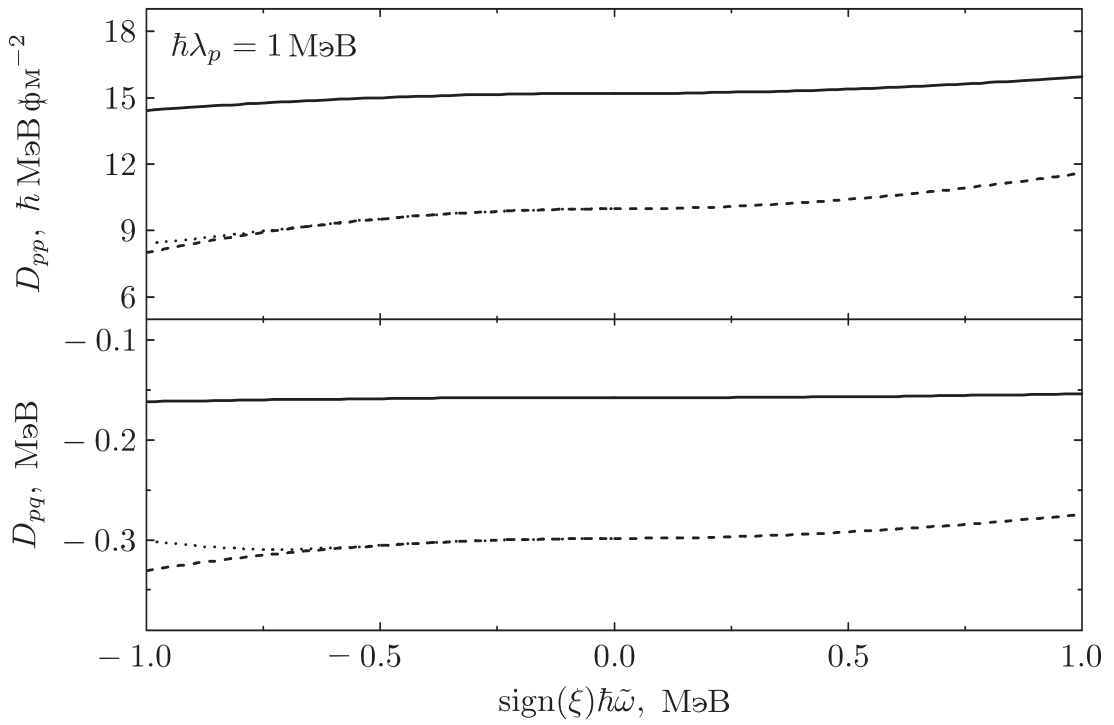

б

Рис. 4. Зависимость асимптотических коэффициентов диффузии от асимптотической частоты гармонического и перевернутого осциллятора при $\mu=448 m_{0}, T=1 \mathrm{MэВ} \mathrm{(сплошная} \mathrm{линия)} \mathrm{и} T=0.3$ МэВ (штриховая линия) при асимптотических значениях коэффициента трения $\hbar \lambda_{p}=3 \mathrm{MэB} \mathrm{(а)} \mathrm{и}$ 1 МэВ (б). Результаты, полученные с модифицированными диффузионными коэффициентами $D_{p p}\left(t=1 / s_{+}\right), D_{q p}\left(t=1 / s_{+}\right)$при $T=0.3$ МэВ, показаны пунктирной линией. 


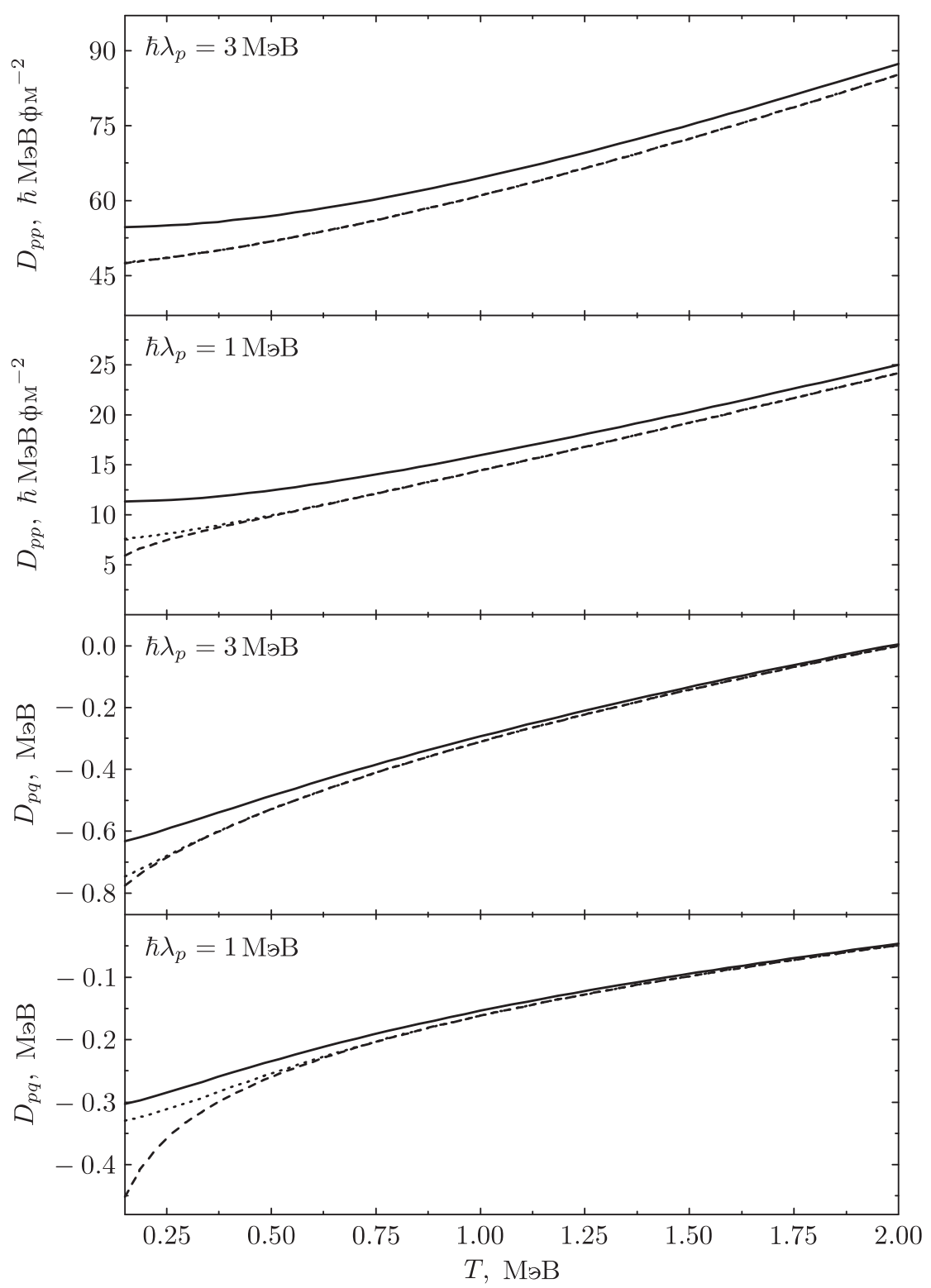

Рис. 5. Зависимости асимптотических коэффициентов диффузии от температуры при $\hbar \widetilde{\omega}=1$ МэВ, $\mu=448 m_{0}$ и асимптотических коэффициентах трения $\hbar \lambda_{p}=1$ и 3 МэВ. Показаны расчеты по микроскопическим формулам (17)-(19) для гармонического осциллятора (сплошная линия, $t=\infty$ ) и перевернутого осциллятора (штриховая линия при $t=\infty$ в (19) и пунктирная линия при $t=1 / s_{+}$в (19)). 
Отсюда видно, что критическая температура уменьшается с ростом $\lambda_{p}$ или с уменьшением $\widetilde{\omega}$. В случае перевернутого осциллятора с $\hbar \widetilde{\omega}=1 \mathrm{MэB} \mathrm{температура} T_{\mathrm{kp}}=$ $0.1 \mathrm{MэВ} \mathrm{и} 0.05 \mathrm{MэВ} \mathrm{при} \hbar \lambda_{p}=1$ и 3 МэВ соответственно. Нефизического поведе-

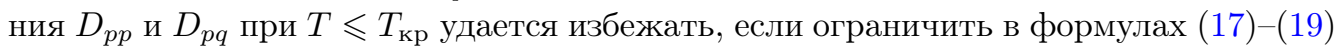
верхние пределы интегрирования по $\tau^{\prime}$ и $\tau^{\prime \prime}$ характерным временем $t=1 / s_{+}$нахождения системы в точке максимальной близости от вершины барьера (характерным временем $t=1 / s_{+}$изменения модуля среднего значения коллективной координаты или импульса в $e$ раз), $\delta p=s_{+} \delta q$. В этом случае при низких температурах значения $D_{p p}$ и $D_{p q}$ для перевернутого осциллятора близки к значениям $D_{p p}$ и $D_{p q}$ для гармонического осциллятора. Отметим, что данная модификация формул не влияет на значения $D_{p p}$ и $D_{p q}$ в области больших температур $T>T_{\text {кр }}$, так как в этом случае характерное время $1 / \gamma$ выхода на асимптотику диффузионных коэффициентов меньше, чем время $t=1 / s_{+}$.

3.2. Проницаемость параболического барьера. Для расчета проницаемости параболического барьера слева направо использована следующая формула [16]:

$$
P(t)=\frac{\rho(t)-\left.\rho(t)\right|_{t=0}}{1-\left.\rho(t)\right|_{t=0}}
$$

где величина

$$
\rho(t)=\frac{1}{\sqrt{2 \pi \sigma_{q q}(t)}} \int_{0}^{\infty} \exp \left[-\frac{(q-\langle q(t)\rangle)^{2}}{2 \sigma_{q q}(t)}\right] d q
$$

учитывает долю гауссовского пакета справа от барьера в момент времени $t$, а нормировочный множитель $1-\left.\rho(t)\right|_{t=0}$ определяет долю начального гауссовского пакета слева от барьера в момент времени $t=0$, так как небольшая часть гауссовского пакета находится справа от барьера. В расчетах $\rho(t)$ и соответственно $P(t)$ использованы $\langle q(t)\rangle$ и $\sigma_{q q}(t)$, рассчитанные с различными наборами диффузионных коэффициентов. Из рис. 6 и рис. 7 видно, что при $\hbar \lambda_{p} \geqslant 1$ МэВ трение мешает прохождению волнового пакета. При относительно высоких температурах ( $T=1 \mathrm{MэB}$ ) значения проницаемости $P=P(\infty)$, рассчитанные с помощью зависящих от времени микроскопических коэффициентов диффузии для перевернутого осциллятора, оказываются приблизительно в 1.5 и в 3-4 раза большими при $\hbar \lambda_{p}=1 \mathrm{MэВ} \mathrm{и} \hbar \lambda_{p}=3$ МэВ соответственно, чем проницаемость, рассчитанная с помощью феноменологического коэффициента $(29)$ при $D_{p q}=0$. Такая тенденция сохраняется и при низких температурах ( $T=0.3 \mathrm{MэB).} \mathrm{Это} \mathrm{объясняется} \mathrm{тем,} \mathrm{что} \mathrm{в} \mathrm{рассматриваемом} \mathrm{диапазоне}$ температур значение асимптотического микроскопического коэффициента диффузии по импульсу больше, чем его феноменологический аналог. На рис. 1 видно, что асимптотическое значение для коэффициента диффузии по импульсу устанавливается после начального всплеска, и, поскольку проницаемость является интегральной характеристикой, ее значение оказывается бо́льшим при использовании зависящих от времени микроскопических коэффициентов диффузии, чем при использовании асимптотических коэффициентов диффузии. Расчеты с микроскопическими асимптотическими коэффициентами диффузии для гармонического осциллятора и модифицированными коэффициентами диффузии для перевернутого осциллятора дают 


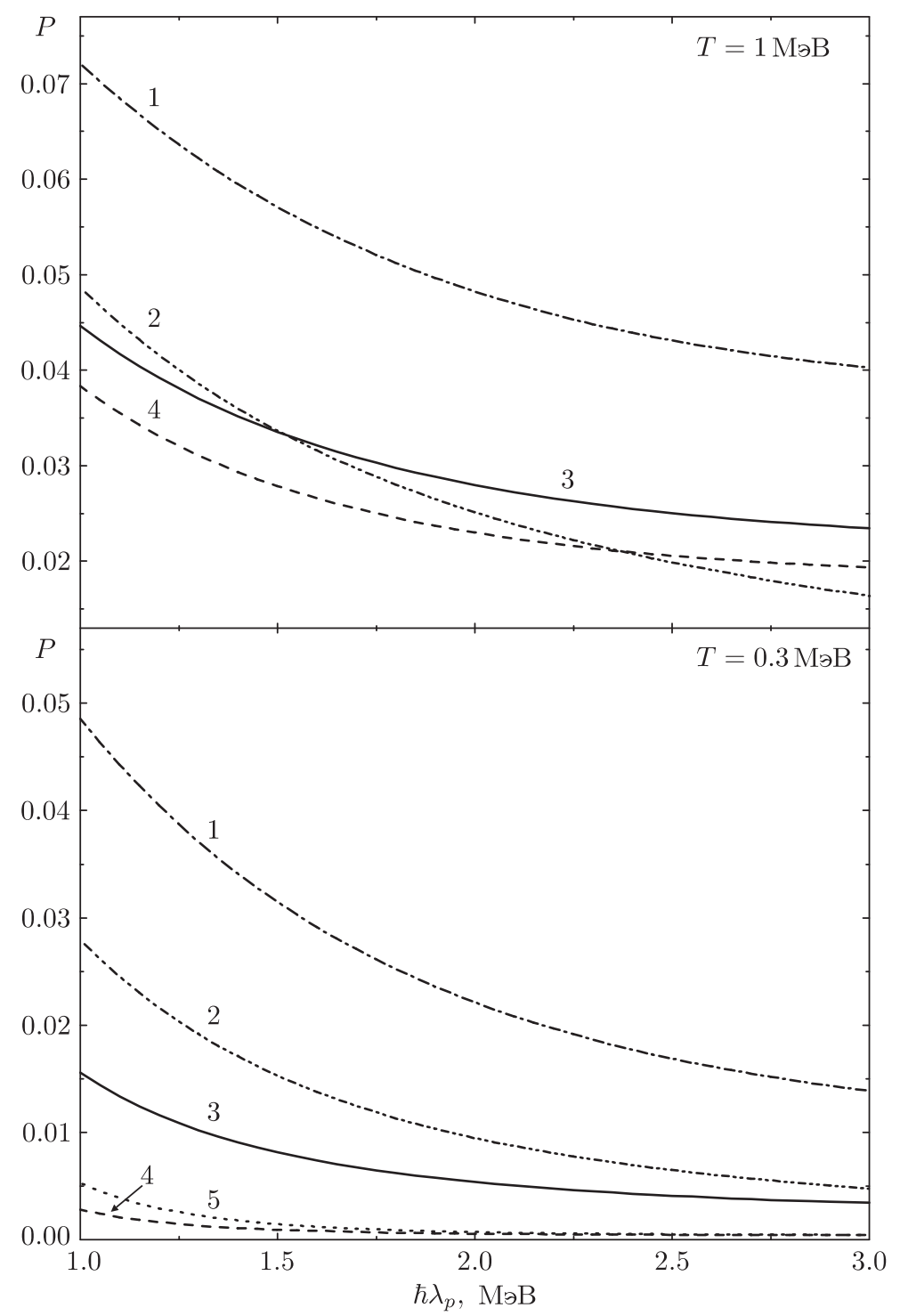

Рис. 6. Зависимость проницаемости $P(\infty)$ параболического барьера от асимптотического коэффициента трения $\lambda_{p}=\lambda_{p}(\infty)$ при $\mu=448 m_{0}, \hbar \widetilde{\omega}=$ 1 МэВ и $\hbar \lambda_{p}=1$ МэВ для $T=1$ МэВ и $T=0.3$ МэВ. В расчетах использованы микроскопические коэффициенты диффузии $D_{p p}(t), D_{q p}(t)(17)-(19)$ (линия 1), асимптотические коэффициенты диффузии $D_{p p}(\infty), D_{p q}(\infty)(25)-(27)$ (линия 4), модифицированные коэффициенты диффузии $D_{p p}\left(t=1 / s_{+}\right)$, $D_{p q}\left(t=1 / s_{+}\right)$(линия 5$)$ для перевернутого осциллятора. Линия 2 - результаты, полученные с феноменологическим коэффициентом диффузии $D_{p p}^{\mathrm{cl}}(29)$ при $D_{p q}=0$, линия $3-D_{p p}(\infty)$ и $D_{p q}(\infty)(25)-(27)$ для гармонического осциллятора. 


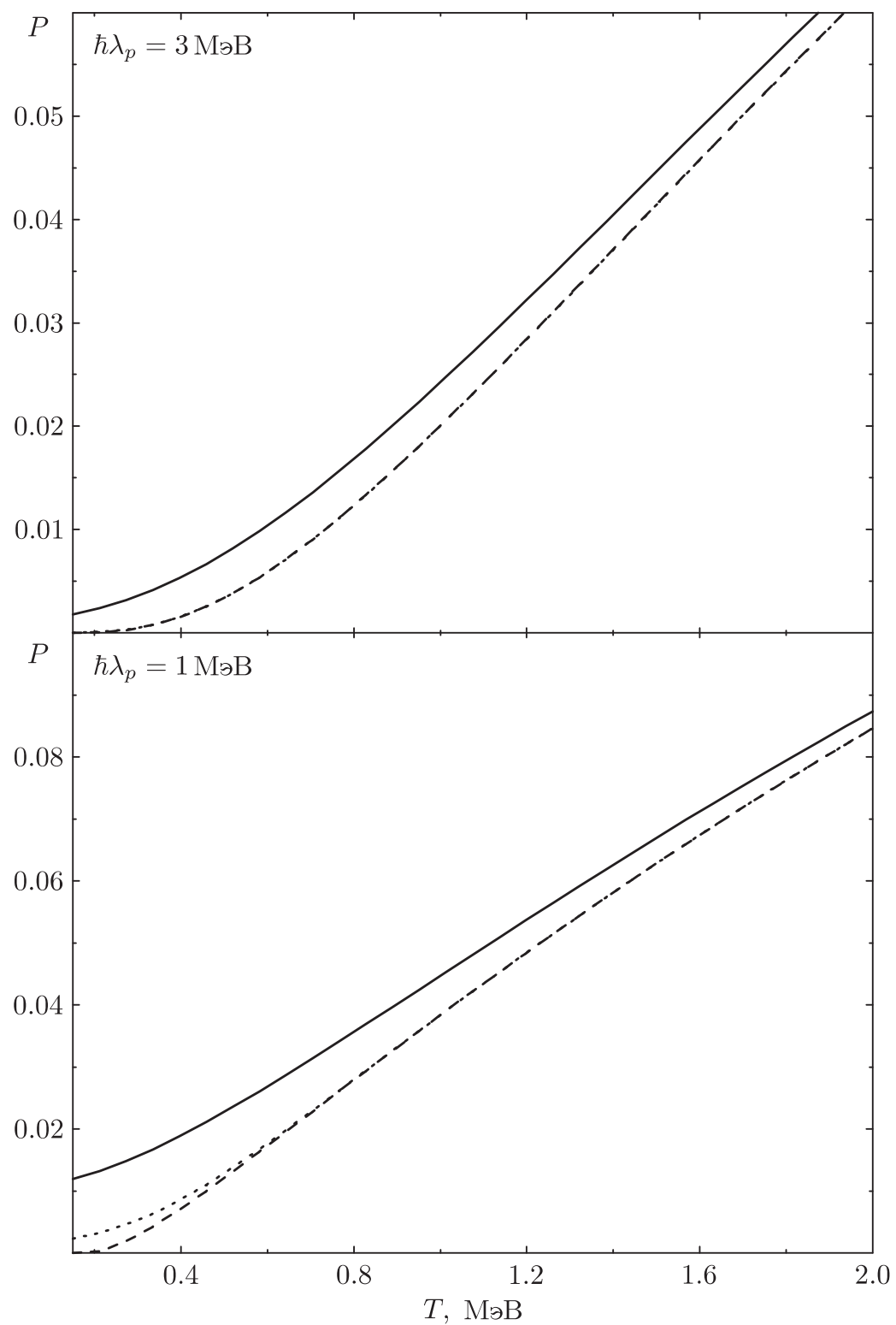

Рис. 7. Зависимость проницаемости $P(\infty)$ параболического барьера от температуры $T$ при $\mu=448 m_{0}, \hbar \widetilde{\omega}=1 \mathrm{MэB}, \hbar \lambda_{p}=3$ МэВ и $\hbar \lambda_{p}=1$ МэВ. В расчетах использованы асимптотические микроскопические коэффициенты диффузии $D_{p p}(\infty), D_{p q}(\infty)(25)-(27)$ (штриховая линия), модифицированные диффузионные коэффициенты $D_{p p}\left(t=1 / s_{+}\right), D_{p q}\left(t=1 / s_{+}\right)$(пунктирная линия) для перевернутого осциллятора и асимптотические микроскопические коэффициенты диффузии $D_{p p}(\infty), D_{p q}(\infty)(25)-(27)$ для гармонического осциллятора (сплошная линия). 
практически одинаковые значения проницаемости во всем рассматриваемом диапазоне коэффициентов трения для относительно высоких температур ( $T=1$ МэВ). Для низких температур $(T=0.3 \mathrm{MэB})$ они отличаются от 5 (при $\hbar \lambda_{p}=1 \mathrm{MэВ)}$ до 8 раз (при $\hbar \lambda_{p}=3$ МэВ). На рис. 7 показано сильное падение $P$ при $T \approx T_{\text {кр и }}$ $\hbar \lambda_{p}=1$ МэВ в расчетах с зависящими от времени микроскопическими коэффициентами диффузии для перевернутого осциллятора.

\section{4. ЗАКЛЮЧЕНИЕ}

С помощью микроскопического гамильтониана полной системы аналитически получена и решена система немарковских уравнений Ланжевена для затухающего перевернутого и гармонического осцилляторов в пределе линейной связи по координате между коллективной и внутренней подсистемами. Показано, что уравнения движения для коллективной подсистемы удовлетворяют квантовому флуктуационнодиссипационному соотношению. Из немарковских уравнений Ланжевена получены локальные по времени уравнения для первого и второго моментов, что позволило найти явные аналитические выражения для коэффициентов трения и диффузии по импульсу и по координате-импульсу, зависящие от времени. Найдены асимптотики транспортных коэффициентов. Полученные нами аналитические формулы могут быть использованы для описания флуктуационно-диссипационной динамики ядерных процессов со сложными потенциалами, например процесса захвата ядер при энергиях около кулоновского барьера.

Сравнительный анализ показал близость абсолютных значений диффузионных коэффициентов затухающего гармонического и перевернутого осцилляторов с одинаковыми коллективными частотами, массами и термостатами. Показано, что асимптотические значения коэффициентов диффузии меняются непрерывно при переходе от гармонического осциллятора к перевернутому осциллятору и наоборот. Предложена модификация диффузионных коэффициентов перевернутого осциллятора при низких температурах в области перехода от режима термической активации к режиму макроскопического квантового туннелирования. Показано, что в пределе сильного затухания проницаемости, рассчитанные с помощью зависящих от времени микроскопических коэффициентов диффузии для перевернутого осциллятора, оказываются в 3-4 раза больше, чем проницаемости, рассчитанные с помощью постоянного феноменологического коэффициента диффузии по импульсу при $D_{p q}=0$.

Благодарности. Работа выполнена при поддержке РФФИ (гранты № 06-0204000, 07-02-00043), Deutsche Forschungsgemeinschaft и Volkswagen-Stiftung.

\section{Список литературы}

[1] Н. Н. Боголюбов, Избранные труды в трех томах, Наукова думка, Киев, 1971.

[2] A. A. Belavin, Ya. B. Zel'dovich, A. M. Perelomov V. S. Popov, ЖЭЭTФ, 56 (1969), 264-274.

[3] Н. Г. Ван Кампен, Стохастические процессы в физике и химии, Высшая школа, М., 1990.

[4] A. O. Caldeira, A. J. Leggett, Phys. A, 121:3 (1983), 587-616; Ann. Phys., 149:2 (1983), $374-456$.

[5] C. W. Gardiner, Quantum Noise, Springer Ser. Synergetics, 56, Springer, Berlin, 1991. 
[6] H. J. Carmichael, An Open System Approach to Quantum Optics, Lecture Notes in Phys., 18, Springer, Berlin, 1993.

[7] Yu. L. Klimontovich, Statistical Theory of Open Systems, V. 1, Fund. Theories Phys., 67, Kluwer, Dordrecht, 1995.

[8] Д. Н. Зубарев, В. Г. Морозов, Г. Рёпке, Статистическая механика неравновесных процессов, Т. 2, Физматлит, М., 2002.

[9] U. Weiss, Quantum Dissipative Systems, Wold Scientific, Singapore, 1999.

[10] V. V. Dodonov, O. V. Man'ko, V. I. Man'ko, J. of Russian Laser Research, 16:1 (1995), 1-56; V.V. Dodonov, V.I. Man'ko, Density Matrices and Wigner Functions of Quasiclassical Quantum Systems, Proc. Lebedev Phys. Inst. of Sciences, 167, ed. A. A. Komar, Nova Science, Commack, NY, 1987.

[11] K. Lindenberg, B. J. West, Phys. Rev. A, 30:1 (1984), 568-582.

[12] H. Grabert, P. Schramm, G.-L. Ingold, Phys. Rep., 168:3 (1988), 115-207; P. Talkner, Ann. Phys., 167:2 (1986), 390-436.

[13] G. W. Ford, J. T. Lewis, R. F. O'Connell, Phys. Rev. A, 37:11 (1988), 4419-4428.

[14] H. Hofmann, D. Kiderlen, Internat. J. Modern Phys. E, 7:2 (1998), 243-274.

[15] Г. Г. Адамян, Н. В. Антоненко, З. Каноков, В. В. Саргсян, ТМФ, 145:1 (2005), 87-101.

[16] G. G. Adamian, N. V. Antonenko, W. Scheid, Phys. Lett. A, 244:6 (1998), 482-488; 260:1-2 (1999), 39-45; Yu. V. Palchikov, G. G. Adamian, N. V. Antonenko, W. Scheid, J. Phys. A, 33:23 (2000), 4265-4276; Phys. A, 316:1-4 (2002), 297-313.

[17] Р. В. Джолос, С. П. Иванова, В. В. Иванов, ЯФ, 40 (1984), 117-125; S. P. Ivanova, R. V. Jolos, Nucl. Phys. A, 530:1 (1991), 232-250.

Поступила в редакцию 26.03.2007, после доработки 18.06.2007 